\section{TGGE-STAR: Primer Design for Melting Analy- sis Using PCR Gradient Gel Electrophoresis}

BioTechniques 32:264-268 (February 2002)

Temperature gradient gel electrophoresis (TGGE) is a type of acrylamide gel electrophoresis that is used to detect point mutations and polymorphisms within PCR products (3). Electrophoresis of the DNA fragments is performed through an increasing linear temperature gradient from the anode to cathode. With increasing temperature, the DNA "melts". Hydrogen bonds are disrupted, and the number of unordered non-helical molecules rises. To prevent both strands from completely falling apart, they are bound by an artificial clamp at one or both ends. Alternatively, the de- naturing gradient can be achieved by increasing the urea concentration within the gel through a process called denaturing gradient gel electrophoresis (DGGE). In the Transgenomic Wave ${ }^{\circledR}$ technique (4), the DNA fragmants also electrophoretically migrate in an increasing temperature environment. TGGE is very fast and sensitive, detecting virtually all heterozygous sequence variations within the PCR product. These qualities should make TGGE the screening method of choice.

However, TGGE is not widely used because the setup is relatively difficult. During our project, the technique exhibited two main difficulties, reducing its applicability. Primarily, the primer design is difficult. Not only must the two primers be compatible with each other but also the melting property of the PCR product must have certain features (7). Secondly, the electrophoretic bands are sometimes fuzzy, and there- fore mutations are not detected (6). This is probably caused by an essentially irreversible conformational change and can be predicted with the computed melting profiles.

The program MELT87.EXE (5) is widely used to calculate melting profiles (Figure 1a) and is available to academic institutions. It has no user interface; instead, it requires the manual preparation of input files and the visualization of the text output with graphic programs. For traditional PCR-primer design, plenty of programs are available. Thus, at the beginning of our project, three programs were involved in the design of the primers: MELT87, a graphical plotting program, and a primer design package. MELT94 is an updated version of MELT87, which contains a graphical display (http://web.mit.edu/osp/www/ melt.html). These three programs were successively run several times to find the best compromise between an opti- 


\section{Benchmarks}

mal melting characteristic and an optimal primer pair.

TGGE-STAR was developed to streamline primer design for TGGE and DGGE so that all steps involved in primer design could be performed within one environment. The software can be downloaded for free from the BioTechniques Software Library (www. BioTechniques.com). The user interface was optimized so that it allows one to design many primers in a short time. To optimize its speed, it was written in ANSI-C to take advantage of fast pointer arithmetic. Because it is a pure MS-

A

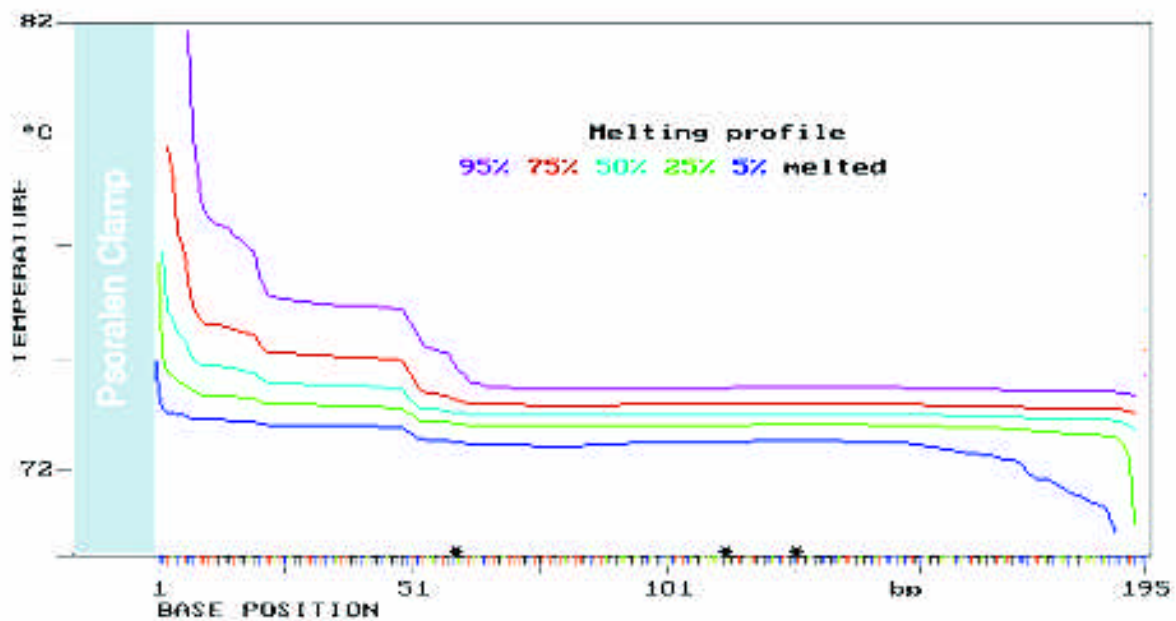

B

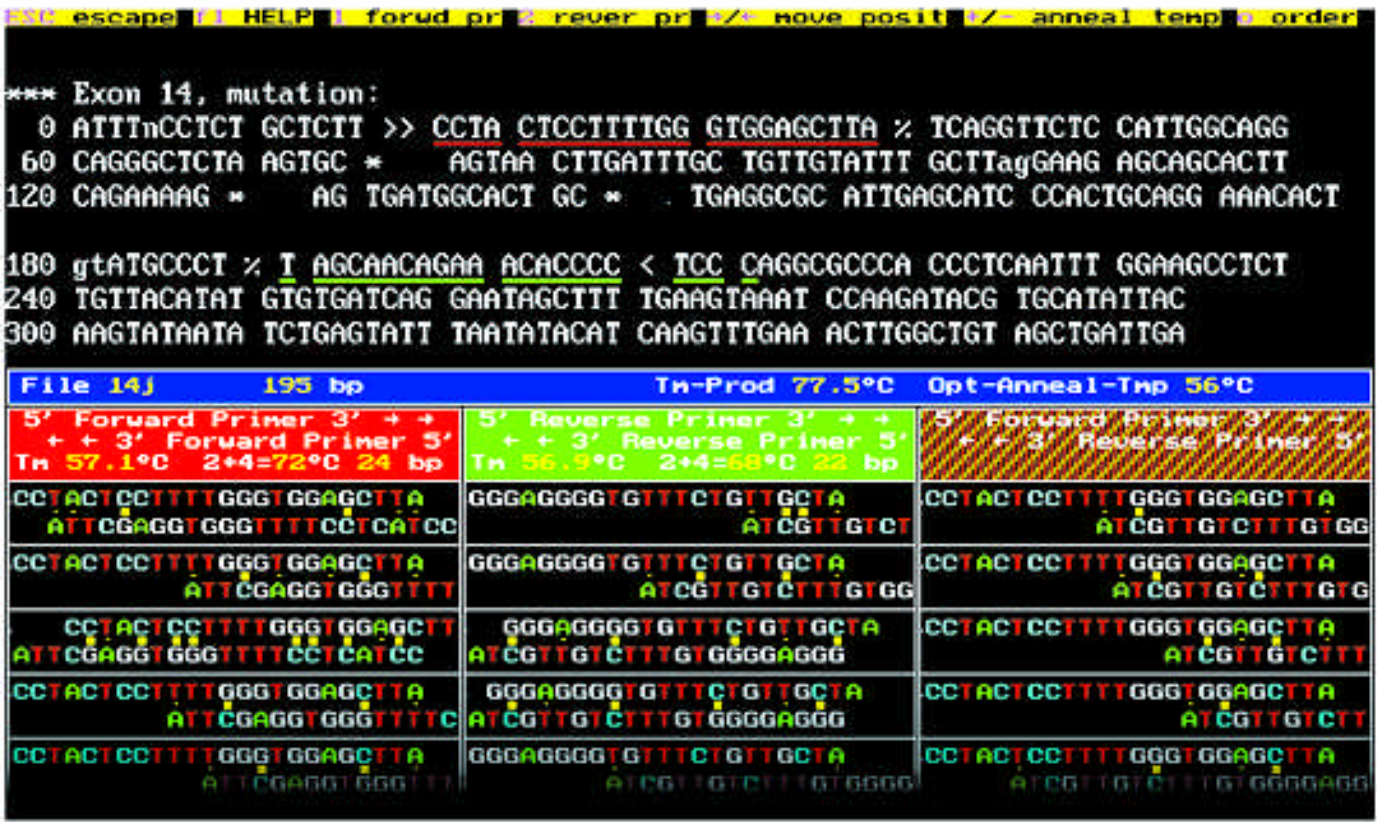

Figure 1. Primer-design unit. Screenshots. (1a) Melting profile. The DNA molecule stretches along the $x$-axis. Each tick on the $x$-axis represents a base pair. The $\mathrm{y}$-axis shows the $\mathrm{T}_{\mathrm{m}}$. The middle line of the group of five "curves" describes the temperature at which a base pair is unordered or helical with an equal probability of 50\%. The other curves correspond to higher or lower probabilities. At the $5^{\prime}$ end (left), the thermostability is artificially increased by a clamp, and the temperature profile ascends. Apart from the clamp, there are two melting domains: from the $5^{\prime}$-terminus to the 50th base pair and from the 50th base pair extending to the $3{ }^{\prime}$-terminus. Generally, the sensitivity in upper melting domains is reduced. However, in this case, the temperature step is not that significant, and all mutations may still be found. Three asterisks above the x-axis mark the positions of the mutations that had been detected with this assay. They had been typed into the sequence text in Figure $1 \mathrm{~b}$ using a text editor. (1b) Primer-design unit. The screen is split into two parts. The upper part of the figure shows the sequence text. The primer binding sites are underlined and can be moved with the cursor keys. Three asterisks were manually inserted at sequence positions where mutations had been identified. The bottom part shows the primer complements. The annealing of identical primers is predicted in the first and second columns, and the annealing of forward with reverse primers is given in the third column. Note that the binding partners are written in opposite directions. The dimers are sorted according to their significance. The goal is to reduce the hydrogen bonds that are drawn as small and big yellow dots. Especially unfavorable are hydrogen bonds at the 3 '-termini. 
DOS program, it runs very fast on native Microsoft ${ }^{\circledR}$ machines and many operating systems that can emulate DOS, such as LINUX or OS/2. The user interface is keystroke-driven and very comfortable. The program consists of two parts: the melting analysis of PCR products (Figure 1a) and the primer optimization (Figure 1b), both of which are closely linked. The calculation of the melting characteristics is automatically performed by MELT87.EXE, which is embedded into TGGE-STAR. The primer optimization part differs from other programs to some degree. First of all, the DNA-sequence file does not require any specific format. Moreover, the sequence is displayed in its original format, preserving all line breaks and comments. This feature greatly increases the orientation in a text that otherwise consists of only four different letters. The primer binding sites are underlined in red and green and can be moved using the arrow keys. Simultaneously, unfavorable pri-

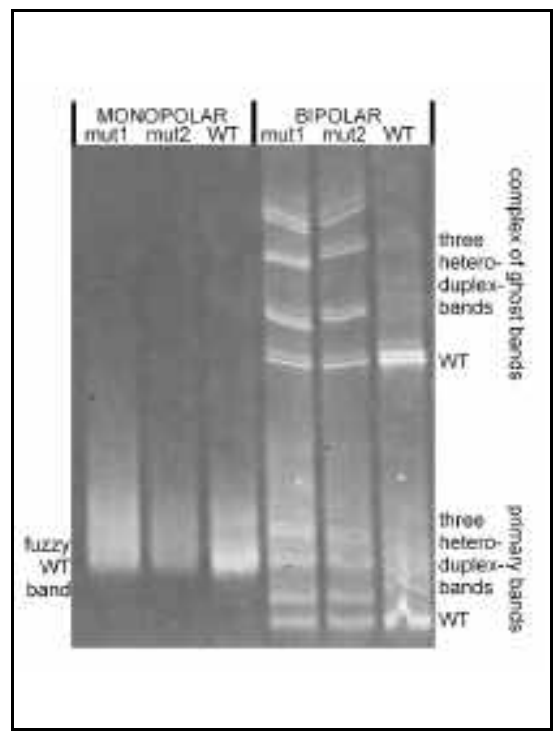

Figure 2. PCR-TGGE bands from two patients carrying mutations in the fibrillin-1 gene com pared to wild-type. The three PCR products were analyzed using traditional monopolar clamping (first three lanes) and bipolar clamping (last three lanes) on the same TGGE gel. The bands in the first three lanes are fuzzy, and the expected heteroduplex bands of the muted probes do not appear. Using bipolar clamping, both mutations can be clearly identified by three heteroduplex bands. The bipolar clamped PCR products (right lanes) migrate slightly further than the monopolar clamped ones (left lanes) because the additional clamp renders the DNA double strand thermally more stable. "Ghost" bands are a frequent phenomenon associated with bipolar clamping. mer self-binding can be displayed in the lower half of the screen. The goal is to minimize primer self-priming. The primer binding sites can also be found automatically. Furthermore, several features were added that proved to be extremely helpful for our project.

Broadened bands are a frequent problem of TGGE and DGGE (6) and were observed in about $20 \%$ of all exons of the NF1 gene (2). It is particularly a problem in genomic DNA analysis because the GC content is lower in introns than in exons. The relative thermic instability of intronic regions can lead to a melting profile that does not decline toward the unclamped end. In our NF1 Project (2), almost all melting profiles with this feature are associated with widened bands, whereas slightly declining profiles are associated with sharp bands. The program supports bipolar clamping that can rescue cases of blurred bands. An explanation of these kinetic phenomena is given in Reference 1 . It is generally believed that despite the increased fuzziness of the bands, sequence variants result in readily detectable band shifts in the gradient. To disprove this assumption, two missed mutations are shown in Figure 2. Both become visible by bipolar clamping.

The superposition of the curves of cumulative probability ( $\bar{p}$-plots) of several fragments can guide the design of multiplex PCR-TGGE essays. This is because the $\bar{p}$-plots provide good estimates for the positions of the bands on the gel. In multiplex-TGGE, it is important that the bands do not obscure each other and that the fragments arrive at their individual $\mathrm{T}_{\mathrm{m}}$ at approximately the same time. The program assists with the fragmentation of the sequence to be analyzed whenever this is necessary due to an unfavorable melting behavior, and one can create an interface to other programs, such as programs for restriction analysis.

\section{REFERENCES}

1.Abrams, E.S., S.E. Murdaugh, and L.S Lerman. 1995. Intramolecular DNA melting between stable helical segments: melting theory. Nucleic Acids Res. 23:2775-2783.

2.Fahsold, R., S. Hoffmeyer, C. Mischung, C. Gille, C. Ehlers, N. Kucukceylan, M. Abdel-Nour, A. Gewies et al. 2000. Minor le- sion mutational spectrum of the entire NF1 gene does not explain. Am. J. Hum. Genet. 66:790-818.

3.Fodde, R. and M. Losekoot. 1994. Mutation detection by denaturing gradient gel electrophoresis (DGGE). Hum. Mutat. 3:83-94.

4.Kuklin, A., A.P. Davis, K.H. Hecker, D.T. Gjerde, and P.D. Taylor. 1999. A novel technique for rapid automated genotyping of DNA polymorphisms. Mol. Cell. Probes 13:239242.

5.Lerman, L.S. and K. Silverstein. 1987. Computational simulation of DNA melting and its application to denaturing gradient gel electrophoresis. Methods Enzymol. 155:482501.

6.Macek, M., Jr., B. Mercier, A. Mackova, P.W. Miller, A. Hamosh, C. Ferec, and G.R. Cutting. 1997. Sensitivity of the denaturing gradient gel electrophoresis technique in detection of known mutations and novel Asian mutations in the CFTR gene. Hum. Mutat. 9:136-147.

7.Poland, D. 1974. Recursion relation generation of probability profiles for specific sequence macromolecules with long-range correlations. Biopolymers 13:1850-1871.

Address correspondence to Dr. Christoph Gille, Institute of Biochemistry, Charité Medical, Faculty of Humboldt University, Monbijoustraße 2a, 10117 Berlin, Germany.e-mail: christoph.gille@ charite.de

Received 7 March 2001; accepted 14 September 2001.

\section{Christoph Gille and Andreas Gille Humboldt University Berlin, Germany}

For reprints of this or any other article, contact Reprints@BioTechniques.com 